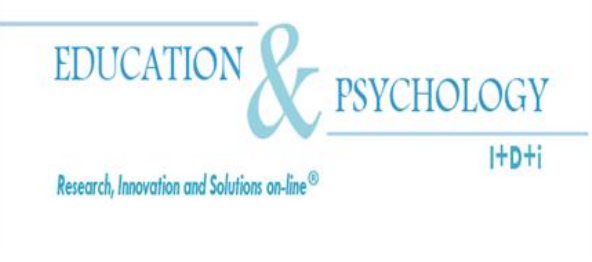

\title{
El papel de la familia en el desarrollo de la comprensión lectora en el cambio de Educación Primaria a Secundaria
}

\section{$M^{a}$ de la Villa Carpio Fernández ${ }^{1}$, $M^{a}$ Cruz Garcia Linares ${ }^{1}$ y Petra Mariscal Ernest ${ }^{2}$}

\author{
${ }^{1}$ Departamento de Psicología de la Universidad de Jaén \\ ${ }^{2}$ Departamento de Psicología de la Universidad de Jaén \\ (Alumna del Master Intervención psicológica en Ámbitos clínicos \\ y de la Salud)
}

\section{España}

Correspondencia: $M^{a}$ de la Villa Carpio Fernández, Departamento de Psicología, edificio C-5. Despacho 145, Universidad de Jaén, Paraje de las Lagunillas s/n, 23071 - Jaén (España)

E-mail: mvcarpio@ujaen.es

C) Education \& Psychology I+D+i and Editorial EOS (Spain) 


\section{Resumen}

Introducción. Este trabajo tiene como objetivo analizar la relación del contexto familiar con la comprensión lectora, en el paso de educación primaria a secundaria.

Método. El trabajo incluye una muestra de 110 alumnos de E.P. y E.S.O con edades comprendidas entre 10 y 15 años. Se evaluaron las prácticas educativas paternas, características sociodemográficas, exposición a la letra impresa y comprensión lectora.

Resultados. Los resultados indican que la comprensión lectora es mayor en alumnos de E.S.O. Se observa una relación entre determinadas variables familiares y la comprensión lectora. Se obtiene que la variable que mejor predice la comprensión lectora es la exposición a la letra impresa.

Discusión y Conclusión. La experiencia con el lenguaje escrito en el hogar familiar, favorece el desarrollo de recursos de comprensión y permite conocer carencias en los-estudiantes adolescentes.

Palabras Clave: contexto familiar, características sociodemográficas, comprensión lectora, exposición a la letra impresa, educación primaria y secundaria. 


\title{
The role of the family in the development of reading comprehension during the transition from Primary to Secondary Education
}

\begin{abstract}
Introduction. The aim of this study is to examine the relationship between family context on reading comprehension at the time of transition from primary to secondary education.
\end{abstract}

Method. The sample consisted of 110 students of primary and secondary education, aged between 10 and 15. It was evaluated parenting practices, socio-demographic characteristics, exposure to print and reading comprehension.

Results. The results indicate that the reading comprehension is higher in the students of secondary education. Secondly, as expected, characteristics of parents correlated positively with reading comprehension. Particularly, the strongest predictor of reading comprehension was exposure to print.

Discussion and Conclusion. Thus, gaining expertise in written language within the family context promotes the development of understanding skills and highlight the possible lacks that students suffers.

Keywords: family background; socio-demographic characteristics; reading comprehension; exposure to print; primary to secondary education 


\section{Introducción}

Desde un punto de vista educativo, la lectura constituye un instrumento básico para el aprendizaje escolar. Aprender a leer implica desarrollar esta habilidad a dos niveles, el reconocimiento de las palabras y la comprensión de la información escrita. Si bien, el reconocimiento de las palabras es un proceso esencial en los primeros años escolares, en años posteriores tiene especial relevancia la comprensión lectora, al constituir una herramienta básica relacionada con el aprendizaje de los alumnos en las diversas materias académicas a través de la cual los estudiantes construyen su conocimiento. Así lo reconoce Barca (2009) cuando indica que un alumno con buen rendimiento escolar es aquél que tiene un buen nivel de comprensión lectora, de igual modo que alguien con mal rendimiento escolar tendrá, con mayor probabilidad, un peor nivel de comprensión lectora. La importancia de la comprensión lectora viene dada por su reconocimiento como índice de calidad lectora (Topping, Samuels y Paul, 2007).

En la actualidad una gran proporción de alumnos de primaria pasan a grados superiores y llegan a la edad adulta sin haber adquirido las habilidades necesarias para comprender lo que leen, presentando dificultad para la localización de las ideas principales, la jerarquización de las mismas y la abstracción de conceptos. La relevancia de la comprensión lectora se pone de manifiesto en el último informe PISA (2010), el cual se centra especialmente en el análisis de las competencias adquiridas por los estudiantes en materia de comprensión lectora. En nuestro país los resultados obtenidos por anteriores informes no son muy favorables al situarnos por debajo del promedio y en el último informe las mejoras han sido escasas (20 puntos en comprensión lectora.

Como todos los procesos evolutivos, el desarrollo de la comprensión lectora está sometido a la influencia de diversos factores que le afectan de forma directa en indirecta (Katzir, Lesaux y Kim, 2009). Si bien, la mayoría de la investigación en esta área se ha centrado en la influencia de factores cognitivos y lingüísticos, recientemente se está produciendo un cambio de perspectiva. Este cambio es debido a la comprobación de que la combinación de todos los factores anteriores explica solo una parte de la varianza de la comprensión lectora, que según el estudio puede ir de 15\% al 70\% (Katzir et al., 2006), lo cual sugiere la necesidad de tener en cuenta otro tipo de variables como las contextuales para conocer el desarrollo de la comprensión lectora. 
El entorno familiar es uno de los factores que inciden en el desarrollo del individuo a lo largo de todas las edades. Su influencia no sólo se circunscribe al contexto más próximo, sino que se extiende a distintos ámbitos, entre ellos al escolar. Los trabajos que demuestran la influencia de los padres sobre los aprendizajes de los niños son muy numerosos (Eccles, Buchanan, Flanagan, Fuligni, Midgley y Yee, 1991; Palacios, Marchesi y Coll, 2004). Sin embargo, específicamente en relación con la lectura son más escasos los estudios llevados a cabo y los realizados se limitan al contexto anglosajon. Con respecto a las variables relacionadas con el contexto familiar, en general, se ha distinguido entre variables próximas (como características socio afectivas del ambiente del hogar) que son experimentadas directamente por el niño y variables distales (como el estatus socioeconómico y otros factores demográficos) que el niño experimenta de forma indirecta (Molfese, Modglin y Molfese, 2003).

La influencia del estatus socioeconómico en la capacidad lectora y los resultados educativos está bien documentada (Arnold y Doctoroff, 2003; Duncan, Yeung, Brooks-Gunn y Smith, 1998; Lee y Burkam, 2002; McLoyd, 1998; Young, Liner, y Brooks-Gunn, 2002). Por ejemplo se ha encontrado que el estatus socioeconómico afecta a las habilidades prelectoras en los años preescolares (McCormick y Mason, 1986). También se conoce que cuanto mayor es el nivel de escolaridad de los padres, mas protagonistas se sienten de su desarrollo y sostienen expectativas de logro más altas con respecto a sus hijos (Palacios, 1988).

En relación con las variables actitudinales y afectivas del contexto familiar destaca el estilo educativo paterno, que si bien en un principio Baumrind (1968) señaló su influencia en la competencia infantil, pronto surgieron investigadores que constataron su relevancia para el ajuste adolescente. Estas dimensiones o prácticas educativas se han relacionado en general con medidas relativas al rendimiento académico (Chao, 2001; Kim y Rohner, 2002; Pérez Díaz, Rodríguez y Sánchez, 2001; Pelegrina, García y Casanova, 2002; Oliva, Parra y Arranz, 2008; Pérez de Pablos, 2003; Peralbo y Fernández, 2003) pero no con medidas más especificas como la comprensión lectora. Aunque Baumrind sólo se había referido a las dimensiones de control y afecto, algunos de los ítems que más discriminan a los padres de los niños más ajustados, se refieren en realidad a la promoción de la autonomía (Lewis, 1981).

Otra dimensión mencionada con frecuencia es el control psicológico, que se refiere a estrategias intrusivas y manipuladoras, como la inducción de culpa o la retirada de afecto, utilizadas por los padres cuando el adolescente muestra un comportamiento que ellos no aprue- 
ban. Se trata de prácticas que violan claramente la individualidad del menor impidiendo el desarrollo de su autonomía e identidad personal. Aunque algunos investigadores han considerado que el control psicológico es el polo opuesto de la promoción o fomento de autonomía, recientemente se ha cuestionado esta consideración, indicando que se trata de dimensiones distintas (Barber, Bean y Erickson, 2002; Silk, Morris, Kanaya y Steinberg, 2003).

Muy interesantes son también las aportaciones de Kerr y Stattin (Kerr y Stattin, 2000; Stattin y Kerr, 2000) sobre la importancia de un aspecto de la comunicación parento-filial como es la revelación (self-disclosure), y que se refiere a la tendencia del adolescente a informar espontáneamente a sus padres acerca de sus actividades en la calle, sus amistades o sus relaciones de pareja, es decir, de lo que hacen cuando sus padres no están presentes. Esta revelación ha mostrado una relación positiva con el ajuste comportamental de los chicos, y puede ser considerada como una estrategia sutil de control parental que previene algunos comportamientos de riesgo (Crouter, Bumpus, Davis y McHale, 2005; Oliva, 2006). Finalmente, Dunn (1993) se ha referido al humor como una dimensión que puede caracterizar las relaciones entre padres e hijos, ya que una actitud parental relajada, alegre y optimista puede crear un clima familiar más favorable que haga más eficaces los intentos socializadores de los padres y promueva el bienestar de niños y adolescentes.

Otra variable relacionada con el contexto familiar que podría mejorar la comprensión lectora y que en la actualidad está siendo analizada, son las prácticas de alfabetización en el hogar, constructo representado por diversos factores interrelacionados como oportunidades para la práctica, promoción de actividades de lectura por los miembros de la familia y motivación (Leseman y Jong, 1998). Otros aspectos incluidos han sido la edad en que el niño empieza a manejar libros, frecuencia de visita a librerías y frecuencia de conductas que interfieren con la lectura (por ejemplo ver televisión). Asimismo se han incluido cuestiones sobre los libros y revistas a los que están suscritos los padres.

El ambiente del hogar puede contribuir de diversas formas en las habilidades lectoras; por un lado, puede proporcionar oportunidades de interacción con la literatura a través de libros disponibles o del modelo que constituyen los padres, o de actividades de lectura realizadas conjuntamente (Anderson y Stokes, 1984; Purcell-Gates, 1996), estas interacciones entre padres e hijos pueden estimular habilidades de lenguaje oral que son importantes para el desarrollo de la comprensión lectora. Hay otras facetas que se refieren a la calidad del apoyo de 
los padres durante las actividades, existe evidencia que sugiere que las diferencias en la calidad de las conductas de los padres durante las actividades de lectura de libros (Whitehurst y Lonigan, 1998) y la frecuencia y calidad de la interacción con los padres en la casa (Hart y Risley, 1995; National Research Council, 2000) contribuye a la disparidad de resultados en la capacidad lectora temprana.

A su vez las distintas variables expuestas han sido relacionadas entre sí. Por ejemplo diversos trabajos han enfatizado la relación entre la exposición a la letra impresa en el hogar y factores como educación o estatus socioeconómico de los padres. (Rashid, Morris y Sevick, 2005). En nuestro contexto el estudio de Jiménez, Rodríguez y Ramírez (2009), llevado a cabo con una muestra española, tiene como uno de sus objetivos determinar si la frecuencia con la que los padres proporcionan apoyo directo a los hijos cuando están aprendiendo a leer produce efectos en sus habilidades lectoras Para ello analiza si las experiencias lectoras en casa y las características socioculturales se asocian con los diversos tipos de dislexia. Sus resultados muestran diferencias entre la conducta de los padres y la educación de los padres en niños con y sin problemas lectores. Las investigaciones más recientes se han ido centrando en las relaciones entre la exposición a la letra impresa y medidas específicas de lectura tales como la comprensión considerada como la habilidad más importante del rendimiento lector en el ambiente escolar (Jong y Leseman, 2001).

Existe evidencia empírica que relaciona de forma específica el ambiente lector del hogar con la comprensión lectora. Los resultados obtenidos por Cipieleswki y Stanovich (1992) indican que las diferencias en comprensión lectora son debidas a la exposición a la letra impresa sugiriendo un papel causal de la exposición a la letra impresa en el desarrollo de la comprensión lectora. Asimismo McBride-Chang, Manis, Seidenberg, Custodio y Doi (1993) defienden que la exposición a la letra impresa medida a través del TRT (Test de Reconocimiento de Títulos de Cunnigham y Stanovich, 1991) predice la comprensión lectora tanto en lectores normales como en lectores con dificultades.

Los hallazgos de Aram y Levin $(2001,2004)$ con niños de bajo estatus socioeconómico de Israel, indican que la calidad de la mediación materna influye en el desarrollo de las habilidades lectoras (entre las que se encuentra la comprensión) en los primeros años escolares. En el estudio llevado a cabo por Rashid et al., (2005) con una muestra de niños con problemas 
lectores se halló una moderada correlación entre las prácticas de lectura de los padres y la comprensión lectora de los niños.

Como podemos observar la mayoría de estos estudios se han centrado en la influencia de las prácticas de alfabetización familiar en el desarrollo lector de niños de corta edad. Según la investigación realizada por Jong y Leseman (2001) la relación entre el ambiente en casa y la comprensión lectora cambia a través del tiempo, en concreto, la relación de ciertas características del ambiente de casa con la comprensión lectora aumentan de primer a tercer grado. Además existe evidencia de que los niños mayores y los adolescentes presentan menos actitudes positivas y menor motivación para la lectura académica y de ocio que los niños más jóvenes y, que, por tanto, leen menos (Eccles, Adler, Futterman, Goff, Kaczala, y Meece, 1983; Jacobs, Lanza, Osgood, Eccles, y Wigfield, 2002; Kush y Watkins, 1996; McKenna, Kear y Ellsworth, 1995; Wigfield, Eccles, Maciver, Reuman, y Midgley, 1991). Sin embargo el apoyo de los padres a la lectura continúa siendo importante en la adolescencia, aunque en este periodo el apoyo sea distinto al que se ofrece a los niños de menor edad (Klauda, 2009). Por ello, la adolescencia resulta un interesante periodo evolutivo para examinar la influencia potencial de los padres en la motivación y los hábitos de lectura de los hijos. Asimismo resulta necesario ampliar el conocimiento sobre la relación entre las prácticas literarias en el hogar y las habilidades lectoras en contextos culturales y lingüísticos diferentes (Kim, 2009).

\section{Objetivos}

Por tanto, en base a lo anteriormente expuesto, este estudio se centra en analizar la influencia de variables sociodemográficas y prácticas educativas junto con el ambiente alfabetizador del hogar en la comprensión lectora de los jóvenes en el paso de la educación primaria a la educación secundaria, un momento especialmente crítico en el rendimiento escolar. Tal y como señalan Eccles et al., (1993) en el comienzo de la adolescencia los jóvenes experimentan múltiples cambios individuales y socio-ambientales que pueden afectar su rendimiento académico. Estos cambios incluyen la emergencia de la pubertad, la transición escolar, el descenso de la motivación académica, la disminución de la dependencia y el incremento del conflicto con los padres. Así pues el comienzo de la adolescencia coincide con los cambios que supone la transición de etapa educativa (de primaria a secundaria) todo lo cual hace este periodo especialmente interesante para el análisis de variables relacionadas con el aprendizaje escolar. 
En concreto, esta investigación se centra en la comprobación de tres objetivos. El primero de ellos, se centra en el análisis de la evolución de la comprensión lectora en el paso de la educación primaria a la educación secundaria El segundo objetivo, aborda el análisis de las relaciones entre las distintas variables relativas al ambiente familiar (prácticas educativas, variables sociodemográficas y exposición a la letra impresa) y la comprensión lectora. Por último, se analizarán cuales de estas variables familiares predicen en mayor medida la comprensión lectora de los adolescentes.

\section{Método}

\section{Participantes}

La muestra estuvo compuesta por un total de 110 estudiantes escolarizados en cuatro niveles educativos, $5^{\circ}$ y $6^{\circ}$ de Educación Primaria (16.4\% y $27.3 \%$, respectivamente) y $1^{\circ}$ y $2^{\circ}$ de Enseñanza Secundaria Obligatoria (25.5\% y 30.9\%, respectivamente), con edades comprendidas entre los 10 y 15 años de edad $(\mathrm{M}=12.63 \mathrm{SD}=1.4)$. Los participantes pertenecían a dos centros educativos públicos de un pueblo de Jaén. (España) La distribución conforme a la variable género fue 55 chicas y 55 chicos, $50 \%$ en ambos casos.

\section{Procedimiento}

Con la finalidad de acceder a los centros educativos se contactó con el equipo directivo y se les explicó la finalidad del estudio, una vez conseguido el consentimiento de los centros, se elaboró una carta dirigida a los padres en la que se explicaba brevemente los objetivos del estudio y se solicitaba el consentimiento para llevar a cabo la pasación de pruebas a sus hijos. Los alumnos fueron evaluados al final del curso, a lo largo del mes de mayo.

\section{Instrumentos}

Estilo parental (Oliva, Parra, Sánchez-Queija y López, 2007). Es una escala que incluye 41 ítems referidos a la percepción que el adolescente tiene del estilo parental de sus padres que eran puntuados a lo largo de una escala tipo Likert de 6 puntos. El instrumento incluye las siguientes sub-escalas: afecto y comunicación (8 ítems, por ejemplo: "Me siento apoyado y comprendido por él/ella" alfa de cronbach $=.88$ ), promoción de autonomía (8 ítems: "Me anima a que tome mis propias decisiones" alfa $=.83$ ), control conductual (6 ítems: "Pone 
límites a la hora a la que debo volver a casa" alfa $=.76$ ), control psicológico ( 8 ítems: "Me hace sentir culpable cuando no hago lo que quiere" alfa $=.80$ ), revelación ( 5 ítems: "Le cuento lo que hago en mi tiempo libre" alfa= .83) y humor (6 ítems: "Suele bromear conmigo" alfa $=.82)$.

Cuestionario de estatus sociodemográfico. El cuestionario consta de 13 de preguntas que miden características socioeconómicas y culturales del ambiente familiar de los alumnos, basadas en el cuestionario elaborado por Burgaleta, Fernández y Martínez Arias (1985), Índice de Características de Status (I.C.S.) que incluye preguntas referidas a la estructura familiar, número de hermanos, profesión y nivel de estudios de los padres e implicación de los padres en el contexto escolar.

Autoinforme sobre la lectura. El cuestionario consta de 12 preguntas de diferente alternativa de respuesta y está basado en el elaborado por Carpio, Defior, Jiménez y Valencia (2008). La primera pregunta mide la motivación hacia la lectura (¿te gusta leer?). Las preguntas 2, 3, 4 y 5 fueron adaptadas del cuestionario de Spear-Swerling (2006) y Baker y Wigfield (1999) con el objetivo de medir la frecuencia con la que los alumnos leen (frecuencia de lectura, lectura de revistas o libros por diversión fuera de la escuela, libros y/o revistas leídas en la última semana, frecuencia con la que leen libros o revistas por diversión fuera del colegio). Las siguientes cuatro preguntas tratan de medir el acceso a materiales de lectura en casa (compra de libros de lectura, compra de revistas y frecuencia con la que compran el periódico). Las cuatro últimas preguntas han sido adaptadas del trabajo de Stanovich y West (1989) con el objetivo de comprobar el conocimiento sobre los títulos de libros/revistas y autores que los niños han leído (libros favoritos leídos, revistas favoritas leídas, autores recordados de los libros leídos y nombre de los autores de los libros que desean leer).

Comprensión lectora. Se utilizó la prueba de comprensión de textos de la Batería PROLEC-SE, "Evaluación de los procesos lectores en alumnos de tercer ciclo de Educación" (Ramos Sánchez, 2007). Este instrumento se compone de seis pruebas diferentes de diversa dificultad, dirigidas a apreciar procesos diferentes: emparejamiento dibujo-oración, comprensión de textos, estructura de un texto, lectura de palabras, lecturas de pseudopalabras y signos de puntuación. En nuestro estudio solo se utiliza la prueba de comprensión de textos, compuesta por dos textos cuyos títulos eran "Los papúes australianos" y "Los esquimales". Esta prueba requiere que el estudiante lea dos textos y posteriormente responda por escrito a diez 
preguntas de cada uno de ellos diferenciando entre preguntas inferenciales y preguntas literales. Las cuestiones literales son aquellas preguntas cuya respuesta aparece de forma explícita en el texto. Las cuestiones inferenciales son aquellas que no aparecen explícitas en el texto pero que pueden deducirse de él y demuestran una total comprensión de la situación. La prueba ofrece número total de aciertos/errores en los dos textos y también el número de aciertos/errores diferenciando preguntas inferenciales y preguntas literales.

\section{Análisis Estadístico}

En primer lugar se utilizaron análisis de varianza univariados para ver la evolución de la comprensión lectora en el paso de la educación primaria a la educación secundaria. En segundo lugar, se utilizaron correlaciones bivariadas mediante el coeficiente de correlación de Sperman para conocer las relaciones entre las variables familiares y la comprensión lectora. Finalemente, se realizaron análisis de regresión lineal para comprobar qué variables familiares predicen en mayor medida la comprensión lectora. Para todos los análsis se utilizó el programa estadístico SPSS.

\section{Resultados}

En relación con el primer objetivo, que suponía comprobar la posible existencia de diferencias en comprensión lectora en el último ciclo de Educación Primaria y el primer ciclo de Educación Secundaria, se realizaron análisis de varianza univariados utilizando como variable independiente el curso académico $\left(5^{\circ}, 6^{\circ}\right.$ de primaria y $1^{\circ}$ y $2^{\circ}$ de educación secundaria) y como variables dependientes la comprensión lectora (número total de aciertos en la prueba, número de aciertos en preguntas literales y número de aciertos en preguntas inferenciales).

En relación con la comprensión lectora global, el ANOVA reveló diferencias significativas en función de los cursos F $(3,109)=24.610$ p<.000. Las comparaciones realizadas, utilizando la prueba de Bonferroni, muestran que la puntuación en comprensión lectora de los alumnos de $5^{\circ}$ de E. P. es significativamente menor que la de los alumnos de $6^{\circ}(p<.000), 1^{\circ}$ de ESO (p<.000) y $2^{\circ}$ de ESO (p<.000), a su vez, los alumnos de $6^{\circ}$ y $1^{\circ}$ de ESO se diferencian de los alumnos de $2^{\circ}$ de ESO p<.000 y p<.022, respectivamente, ver la Figura 1.

En relación con la evaluación de la comprensión lectora en preguntas literales, el ANOVA revela diferencias significativas en función del curso $F(3,109)=20.202$ p <.000. Las 
comparaciones realizadas, utilizando la prueba de Bonferroni, muestran que la puntuación en comprensión lectora de los alumnos de $5^{\circ}$ de E.P. es significativamente menor que la de los alumnos de $6^{\circ}(\mathrm{p}<.000), 1^{\circ}$ de ESO $(\mathrm{p}<.000)$ y $2^{\circ}$ de ESO (p<.000), los alumnos de $6^{\circ}$ se diferenciación de los alumnos de $2^{\circ}$ ESO p<.004, tal y como vemos en la Figura 1.

En relación con la evaluación de la comprensión lectora en preguntas inferenciales, el ANOVA revela diferencias significativas en función del curso $F(3,109)=23.380$ p <.000 Las comparaciones realizadas, utilizando la prueba de Bonferroni, muestran que la puntuación en comprensión lectora de los alumnos de $5^{\circ}$ de E.P. es significatimente menor que la de los alumnos de $6^{\circ}(\mathrm{p}<.002), 1^{\circ}$ de ESO (p<.000) y $2^{\circ}$ de ESO (p<.000), a su vez, los alumnos de $6^{\circ}$ y $1^{\circ}$ de ESO se diferencian de los alumnos de $2^{\circ}$ de ESO $p<.004$ y $p<.02$, respectivamente, tal y como podemos ver en la Figura 1.

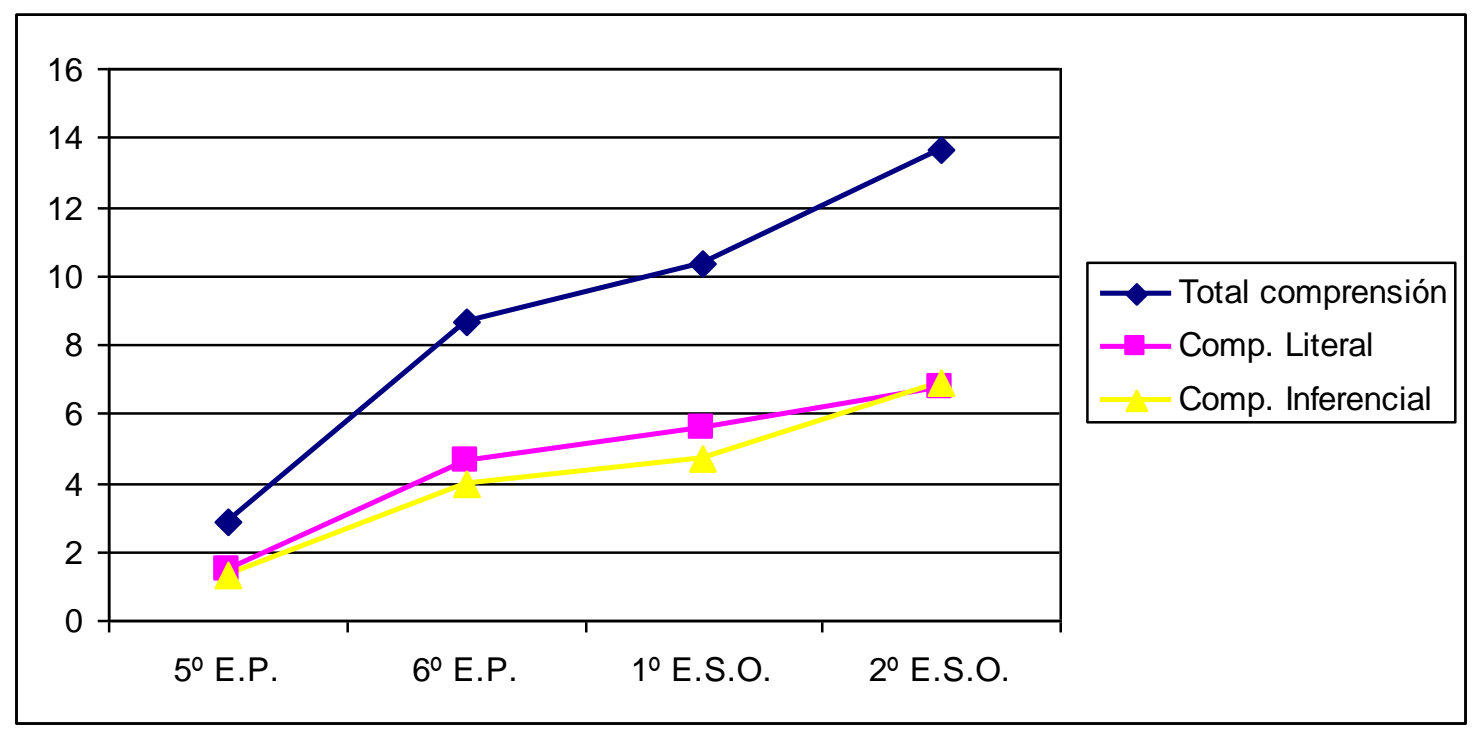

Figura 1. Puntuaciones medias en comprensión lectora en los diferentes cursos.

Para la comprobación del segundo objetivo, es decir analizar la posible existencia de relaciones entre las variables familiares y la comprensión lectora, analizamos las correlaciones bivariadas existentes entre dichas variables mediante el coeficiente de correlación de Spearman, tal y como podemos ver en la Tabla 1. 
Tabla 1. Correlaciones entre las medidas familiares y la comprensión lectora

\begin{tabular}{l|lll}
\hline & $\begin{array}{l}\text { Comprensión } \\
\text { Literal }\end{array}$ & $\begin{array}{l}\text { Comprensión } \\
\text { inferencial }\end{array}$ & $\begin{array}{l}\text { Comprensión } \\
\text { total }\end{array}$ \\
\hline 1. Afecto & -.146 & -.159 & -.153 \\
2.Independencia & -.126 & -.162 & -.150 \\
3. Control conductual & -.044 & .078 & .026 \\
4. Control psicológico & $-.204(*)$ & -.176 & $-.200(*)$ \\
5. Revelación & -.052 & -.005 & -.027 \\
6. Humor & -.083 & -.167 & -.120 \\
7. Exposición letra impresa & $.403(* *)$ & $.504(* *)$ & $.473(* *)$ \\
8. Estudios madre & $.230(*)$ & $.339(* *)$ & $.302(* *)$ \\
9. Estudios padre & $.237(*)$ & $.298(* *)$ & $.284(* *)$ \\
10. Número hermanos & -.150 & -.103 & -.152 \\
11.Número de habitaciones & .128 & .156 & .143 \\
12. Estructura familiar & -.044 & -.032 & -.036 \\
13. Tutorías & $.200(*)$ & $.261(* *)$ & $.247(*)$ \\
\hline
\end{tabular}

$* \mathrm{p}<0.05$ (bilateral) $* * \mathrm{p}<0.01$ (bilateral).

Las variables que correlacionan con la medida de comprensión lectora total y comprensión en preguntas literales son las mismas, es decir: el control psicológico negativamente, y la exposición a la letra impresa, los estudios de la madre y del padre y la asistencia a tutorías positivamente. Con la medida de comprensión lectora inferencial se relacionan las siguientes variables: exposición a la letra impresa, estudios de la madre y estudios del padre y la asistencia a tutorías.

Por último, para conocer el peso estadístico de las diferentes variables familiares en la comprensión lectora de los estudiantes se realizaron tres análisis de regresión lineal, el primero para la comprensión lectora literal, el segundo para la inferencial y el tercero para la comprensión lectora global (literal e inferencial).

El modelo no resultó significativo para la comprensión literal, pero si para la comprensión inferencial y global. Con respecto a la comprensión inferencial, la variable que resultó con mayor capacidad predictiva en la comprensión lectora fue la exposición a la letra impresa $(\beta=.224, \mathrm{p}<.000)$, ver Tabla 2 . En cuanto a la comprensión global (literal e inferencial) de nuevo la variable con mayor capacidad predictiva fue la exposición a la letra impresa $(\beta=$ $.175, \mathrm{p}<.05)$, ver Tabla 3 . 
Tabla 2. Coeficientes de las variables familiares para el análisis de la regresión lineal en la comprensión lectora inferencial

\begin{tabular}{|c|c|c|c|c|c|c|}
\hline \multirow[t]{2}{*}{ Modelo } & & \multicolumn{2}{|c|}{$\begin{array}{l}\text { Coeficientes no estan- } \\
\text { darizados }\end{array}$} & $\begin{array}{l}\text { Coeficientes } \\
\text { estandarizados }\end{array}$ & $t$ & Sig \\
\hline & & $\beta$ & Error tip. & \multicolumn{3}{|l|}{$B$} \\
\hline \multirow[t]{14}{*}{1} & Constante & -.448 & 2.042 & & -.219 & .827 \\
\hline & Estructura familiar & -.885 & 1.029 & -.089 & -.861 & .392 \\
\hline & $\mathrm{N}^{\mathrm{a}}$ hermanos & .023 & .270 & .008 & .084 & .933 \\
\hline & $\mathrm{N}^{\circ}$ habitaciones & -.047 & .106 & -.048 & -.443 & .659 \\
\hline & Estudios padres & .127 & .461 & .041 & .277 & .783 \\
\hline & Estudios madre & .518 & .449 & .175 & 1.153 & .252 \\
\hline & Asisten. Tutorías & .322 & .241 & .134 & 1.338 & .185 \\
\hline & Exposición letra impresa & .224 & .052 & .404 & 4.318 & .000 \\
\hline & Afecto & .064 & .064 & .280 & 1.009 & .316 \\
\hline & Independencia & -.037 & .063 & -.151 & -.586 & .559 \\
\hline & Control conductual & .070 & .048 & .227 & 1.457 & .149 \\
\hline & Control psicológico & -.023 & .048 & -.062 & -.467 & .642 \\
\hline & Revelación & -.002 & .049 & -.008 & -.045 & .964 \\
\hline & Humor & -.097 & .069 & -.322 & -1.401 & .165 \\
\hline
\end{tabular}

Tabla 3. Coeficientes de las variables familiares para el análisis de regresión lineal en la comprensión global

\begin{tabular}{|c|c|c|c|c|c|c|}
\hline \multirow[t]{2}{*}{ Modelo } & & \multicolumn{2}{|c|}{$\begin{array}{l}\text { Coeficientes no estandari- } \\
\text { zados }\end{array}$} & $\begin{array}{l}\text { Coeficientes } \\
\text { estandarizados }\end{array}$ & $T$ & Sig \\
\hline & & $\beta$ & Error tip. & \multicolumn{3}{|l|}{$\beta$} \\
\hline \multirow[t]{14}{*}{1} & Constante & 1.306 & 3.982 & & .328 & .744 \\
\hline & Estructura familiar & -1.527 & 2.006 & -.082 & -.761 & .449 \\
\hline & $\mathrm{N}^{\circ}$ hermanos & .073 & .526 & .014 & .139 & .890 \\
\hline & $\mathrm{N}^{\circ}$ habitaciones & -.073 & .206 & -0.40 & -.355 & .723 \\
\hline & Estudios padres & .515 & .898 & .088 & .573 & .568 \\
\hline & Estudios madre & .596 & .876 & .107 & .681 & .498 \\
\hline & Asisten. Tutorías & .570 & .470 & .126 & 1.213 & .228 \\
\hline & Exposición letra impresa & .399 & .101 & .382 & 3.943 & .000 \\
\hline & Afecto & .101 & .124 & .232 & .808 & .421 \\
\hline & Independencia & -.067 & .123 & -.145 & -.542 & .589 \\
\hline & Control conductual & .098 & .094 & .169 & 1.043 & .300 \\
\hline & Control psicológico & -.069 & .094 & -.100 & -.733 & .466 \\
\hline & Revelación & -.013 & .095 & -.023 & -.133 & .894 \\
\hline & Humor & -.122 & .135 & -.215 & -.903 & .369 \\
\hline
\end{tabular}

\section{Discusión y conclusiones}

En relación con el primer objetivo, los resultados obtenidos demuestran que la comprensión lectora aumenta de forma significativa a lo largo de los cursos analizados. Este resultado se comprueba tanto en la medida global de comprensión como en las medidas de comprensión referidas a preguntas literales y a preguntas inferenciales. Este resultado coincide 
claramente con el obtenido por García Madruga y Fernández Corte (2008) en cuyo estudio se compara un grupo de alumnos de $1^{\circ}$ de ESO con un grupo de alumnos de $4^{\circ}$ de ESO. Dichos autores explican este incremento aludiendo a un aumento en la eficacia de los procesos superficiales de lectura, lo que libera recursos que pueden ser aplicados a la comprensión, y además, por la adquisición y uso de estrategias de comprensión. En nuestro caso, el patrón de resultados se reproduce en las tres medidas de comprensión e indica un aumento significativo en los alumnos de $6^{\circ}$ con respecto a los de $5^{\circ}$, aumento que se mantiene en el primer curso de Educación Secundaria Obligatoria, produciéndose un nuevo incremento en $2^{\circ}$ curso de ESO. Estos resultados implican que las habilidades lectoras continúan aumentando en estas edades más avanzadas.

En cuanto al segundo objetivo planteado, los resultados indican que las variables familiares se asocian con la comprensión lectora. En relación con las variables sociodemográficas, destaca la relación que se pone de manifiesto entre el nivel de estudios de la madre y del padre con la comprensión lectora. Los hijos cuyos padres poseen mayor cantidad de años de escolaridad, especialmente en el caso de las madres, muestran mayor competencia lectora. En este sentido, el informe PISA recoge que, a pesar de las cautelas que deben tenerse para interpretar el impacto del nivel de estudios de los padres en el progreso de los alumnos, las informaciones que se obtienen refuerzan esta relación. Tres datos lo atestiguan fehacientemente: las conclusiones del estudio PISA, los indicadores de la OCDE y las propias comparaciones realizadas en España sobre el nivel de estudios de la población y tasa de finalización de estudios en las distintas Comunidades Autónomas. El informe PISA (OCDE, 2001) señaló que los estudiantes cuyas madres no han terminado los estudios de educación secundaria superior tienen unas puntuaciones en habilidad lectora 44 puntos por debajo de aquellos estudiantes cuyas madres han terminado este tipo de estudios. Cuando se controlan otros factores relacionados con el entorno familiar que también influyen en el rendimiento de los alumnos, cada año adicional de la educación de los padres añade al menos, 4.7 puntos a las puntuaciones de los alumnos. En el caso de España, se añaden 3.6 puntos.

La asistencia a tutorías, que en este caso puede interpretarse como un índice de la implicación de los padres en la educación de sus hijos, se asocia con la comprensión lectora de los hijos. En diversos estudios se ha demostrado que la implicación de los padres en la educación de los hijos favorece el rendimiento académico de los jóvenes (Grolnick y Slowiaczek, 1994), en este estudio se muestra una clara relación con la comprensión lectora. 
En cuanto a las prácticas educativas paternas nuestros resultados indican, coincidiendo con los obtenidos por otros autores (Barber, Olsen y Shagle, 1994; Barber y Harmon, 2002; Oliva et al., 2007) que el control psicológico resulta poco recomendable, puesto que se relaciona con problemas emocionales. Los datos de este estudio indican que, además, el control psicológico aparece asociado de forma negativa con la comprensión lectora total y con la comprensión lectora en las preguntas literales, es decir el control psicológico se asocia con una peor comprensión lectora. Según Mills y Rubin (1998) el control psicológico puede contribuir al desarrollo de un bajo sentido de autoeficacia, al interferir con las oportunidades para ejercitar la competencia y al exponer al niño al rechazo y las valoraciones negativas durante las interacciones con los padres. Si se considera que el control psicológico incluye estrategias tales como la inducción de culpa y la retirada de afecto, se puede entender que este tipo de control se haya asocidado no solo a problemas de ajuste, sino también a una disminución del rendimiento en los hijos (Aunola y Nurmi 2004; Barber y cols., 2002) al dañar la iniciativa y el logro del niño. Así pues, en futuras investigaciones, resultaría interesante comprobar si esta disminución del rendimiento podría estar mediada por la influencia negativa en la comprensión lectora, dato este último que se comprueba en el presente estudio.

Por lo que respecta a la exposición a la letra impresa, los resultados obtenidos muestran claramente su relación con la comprensión lectora, tanto si se considera la puntuación global de la prueba como si se tiene en cuenta la comprensión relativa a preguntas inferenciales y a preguntas literales. Específicamente la exposición a la letra impresa ha sido la variable con mayor capacidad predictiva en la comprensión lectora global y la inferencial. En las preguntas inferenciales los alumnos tienen que realizar una completa comprensión del texto en la que tienen que hacer deducciones y comprender lo que está implícito en el mismo. Los estudiantes que consiguen realizar estas inferencias son aquellos que tienen más conocimientos previos sobre el tema, mientras que los estudiantes que no lo consiguen necesitarán estrategias concretas que le ayuden a obtener una información que, en principio, no aparece en el texto explícitamente. Los estudiantes que leen frecuentemente textos, al margen de los leídos en la escuela, van a desarrollar un vocabulario más complejo así como conocimientos en general (Cunnigham y Stanovich, 1991). El papel de la familia es esencial en este aspecto ya que un ambiente estimulante en el hogar favorece el desarrollo de recursos de comprensión más complejos en los alumnos de estas edades, de esta forma la experiencia con la letra impresa, a través de lecturas en casa conducirá a un crecimiento de la comprensión lectora y de las capa- 
cidades cognitivas relacionadas con ésta. (Echols et al., 1996).

Parece, por tanto, que la medida de exposición a la letra impresa resulta un factor importante que modula las diferencias individuales en las habilidades de lenguaje escrito (Oakhill y Cain, 2004) y cuya evaluación, de forma fiable y válida, puede ser de ayuda para comprender posibles retrasos o carencias en el aprendizaje de los estudiantes, así como un posible factor de intervención para la prevención y corrección de problemas lectores tanto en niños como en adolescentes.

Hay que señalar algunas limitaciones que este trabajo presenta. Una de ellas tiene que ver con el hecho de haber utilizado una única fuente de información, el adolescente, lo que puede haber generado cierto sesgo al aumentar el tamaño de las correlaciones entre las variables estudiadas. En segundo lugar también hay que señalar el pequeño tamaño de la muestra y el hecho de que los sujetos no hayan sido seleccionados al azar. En este sentido, futuras investigaciones deberían obtener información procedente de distintas fuentes (por ejemplo padres y maestros), asi como ampliar la muestra y utilizar un sistema de selección de los sujetos para aumentar su representatividad. Sin embargo este estudio aporta nuevos datos sobre la asociacion entre el contexto familiar y la comprensión lectora, la cual constituye una de las habilidades básicas relacionadas con el aprendizaje de los alumnos, extendiendo dicha asociación al periodo de la adolescencia.

\section{Referencias}

Anderson, A. y Stokes, S. (1984). Social and institutional influences on the development and practice of literacy. En H. Goelman, A. Oberg y F. Smith (Eds.), Awakening to Literacy (pp. 24-37). Portsmouth, NJ: Heinemann.

Aram, D. y Levin, I. (2001). Mother-child joint writing in low SES: sociocultural factors, maternal mediation and emergent literacy. Cognitive Development, 16, 831-852.

Aram, D. y Levin, I. (2004).The role of maternal mediation of writing to kindergartners in promoting literacy in school: a longitudinal perspective. Reading and Writing, 17, 387405.

Arnold, D. y Doctoroff, G. (2003).The early education of socioeconomically disadvantaged children. Annual Review of Psychology, 54, 517-545.

Aunola, K. y Nurmi, J.E. (2005). The role of parenting styles in children's problem behavior. Child Development, 76, 1144-1159. 
Baker, L. y Wigfield, A. (1999). Dimensions of children's motivation for reading and their relations to reading activity and reading achievement. Reading Research Quarterly, 34, 424-477.

Barber, B. K., Bean, R. L., y Erickson, L. D. (2002). Expanding the study and understanding of psychological control. En B.K. Barber (Eds.), Intrusive parenting: How psychological control affects children and adolescents (pp.263-290).Washington, DC: American Psychological Association Press.

Barber, B.K., Olsen, J.E. y Shagle, S.C. (1994). Associations between psychological and behavioral control and youth internalized and externalized behaviors. Ckild Development, $65,1120-1136$.

Barber, B. K. y Harmon, E. L. (2002). Violating the self: Parental psychological control of children and adolescents. En B.K. Barber (Eds.), Intrusive parenting: How psychological control affects children and adolescents (pp. 15-52). Washington, DC: APA.

Barca, A. (2009) Perfiles motivacionales del alumnado de Educación Secundaria y rendimiento académico. Educação Temática Digital 10, 62-84

Baumrind, D. (1968). Authoritarian vs. authoritative parental control. Adolescence, 3, 255272.

Burgaleta, R., Fernández, J y Martínez Arias, M.D. (1985). Índice de Características de Estatus. Madrid: Universidad Complutense.

Carpio, M.V., Defior, S., Jiménez, G. y Valencia, N. (2008). Exposición al lenguaje escrito en niños de educación primaria. En E. Diez-Itza (Eds.), Estudios de Desarrollo del lenguaje y Educación. ICE: Universidad de Oviedo.

Cipielewski, J. y Stanovich, K.E. (1992). Predicting growth in reading ability from children's exposure to print. Journal of Experimental Child Psychology, 54, 74-89.

Chao, R.K. (2001). Extending research on the consequences of parenting style for Chinese Americans and European Americans. Child Development, 72, 1832-1843.

Crouter, A. C., Bumpus, M. F., Davis, K. D. \& McHale, S. M. (2005). How do parents learn about adolescents' experiences? Implications for parental knowledge and adolescent risky behavior. Child Development, 76, 869-882.

Cunningham, A.E. y Stanovich, K.E. (1991). Tracking the unique effects of print exposure in children: Associations with vocabulary, general knowledge, and spelling. Journal of Educational Psychology, 83, 264-274.

Jong, P. y Leseman, P. (2001). Lasting effects of home literacy on reading achievement in school. Journal of School Psychology, 39, 389-414. 
Duncan, G. J.,Yeung, W. J., Brooks-Gunn, J. y Smith, J. R. (1998). How much does childhood poverty affect the life chances of children?. American Sociological Review, 63(3), 406-423.

Dunn, J. (1993). Young children close relationships: Beyond attachment. Newbury Park: Sage.

Eccles (Parsons), J., Adler, T. F., Futterman, R., Goff, S. B., Kaczala, C. M., Meece, J. L., et al. (1983). Expectancies, values, and academic behaviors. In J. T. Spence (Ed.), Achievement and achievement motivation (pp. 75-146). San Francisco, CA: W.H. Freeman.

Eccles, J.S., Buchanan, C.M., Flanagan, C., Fuligni, A., Midgley, C., y Yee, D. (1991). Control versus autonomy during early adolescence. Journal of Social Issues, 47(4), 53 68.

Eccles, J. S., Midgley, C., Wigfield, A., Buchanan, C. M., Reuman, D., Flanagan, C. y MacIver, D. (1993). The impact of stage-environment fit in young adolescents' experiences in school and in families. American Psychologist. 48, 90-101.

Echols, L.D., West, R.F, Stanovich, K.E. y Zher, K.S. (1996). Using children's literacy activities to predict growth verbal cognitive skills: a longitudinal investigation. Journal of Educational Psychology, 88(2), 296-304.

Garcia Madruga, J.A. y Fernández Corte, T. (2008). Memoria operativa, comprensión lectora y razonamiento en la educación secundaria. Anuario de Psicología, 39(1), 133-157.

Grolnick, W. y Slowiaczek, M. (1994). Parents' involvement in children's schooling: A multidimensional conceptualization and motivational model. Child Development, 65, 237252.

Hart, B. y Risley, T. R. (1995). Meaningful differences in the everyday experience of young American children. Baltimore, MD: Brookes.

Jacobs, J. E., Lanza, S., Osgood, D. W., Eccles, J. S. y Wigfield, A. (2002). Ontogeny of children's self beliefs: Gender and domain differences across grades one through 12. Child Development, 73, 509-527.

Jiménez, J.E., Rodríguez, C. y Ramírez, G. (2009). Spanish developmental dislexia: Prevalence, cognitive, profile and home literacy experiences. Journal of Experimental Child Psychology, 103,167-185.

Katzir, T., Kim, Y., Wolf, M., O’Brien, B., Kennedy, B., Lovett, M. y Morris, R. (2006). Reading fluency: The whole is more than the parts. Annals of Dyslexia, 56, 51-82.

Katzir, T., Lesaux, N.K. y Kim, Y.S. (2009). The role of reading self-concept and home literacy practices in fourth grade reading comprehension. Reading and Writing, 22, 261-276. 
Kerr, M. y Stattin, H. (2000). What Parents know, How They Know it, and Several Forms of Adolescent Adjustment: Further Support for a Reinterpretation of Monitoring. Developmental Psychology, 36(3), 366-380.

Kim, Y. (2009). The relationship between home literacy practices and developmental trajectories of emergent literacy and conventional literacy skills for Korean children. Reading and Writing, 22, 57-84.

Kim, K. y Rohner, R. P. (2002). Parental warmth, control, and involvement in schooling:Predicting academic achievement among Korean American adolescents. Journal of Cross-Cultural Psychology, 33, 127-140.

Klauda, S.L. (2009). The Role of Parents in Adolescents' Reading Motivation and Activity. Educational Psychology Review, 21, 325-363

Kush, J. C. y Watkins, M. W. (1996). Long term stability of childrens' attitudes toward reading. Journal of Educational Research, 5, 315-319.

Lee, V. E. y Burkam, D. T. (2002). Inequality at the starting gate: Social background differences in achievement as children enter school. Washington, DC: Economic Policy Institute.

Leseman, P. y Jong, P. (1998). Home literacy: Opportunity, instruction, cooperation and social-emotional quality predicting early achievement. Reading Research Quarterly, 33, 294-318.

Lewis, C.C. (1981). The effects of parental firm control: A reinterpretation of findings. Psychological Bulletin, 90, 547-563.

McBride-Chang, C., Manis, F. R., Seidenberg, M. S., Custodio, R. y Doi, L. M. (1993). Print exposure as a predictor of word reading and reading comprehension in disabled and nondisabled readers. Journal of Educational Psychology, 85, 230-238.

McCormick, C. y Mason, J. (1986). Intervention procedures for increasing preschool children's interest in and knowledge about reading. En W. Teale y E. Sulzby (Eds.), Emergent literacy: Writing and reading, (pp. 90-115). Norwood, NJ: Albex.

McKenna, M.C., Kear, D. J. y Ellsworth, R.A. (1995). Children's attitudes toward reading: A national survey. Reading Research Quarterly, 30, 934-956.

McLoyd, V.C. (1998). Socioeconomic disadvantage and child development. American Psychologist, 53, 185-204.

Mills, R.S. y Rubin K.H. (1998). Are Behavioural and Psychological Control both differentially associated with childhood aggression and social withdrawal? Canadian Journal of Behavioural Science, 30, 132-136 
Molfese, V.J., Modglin, A. y Molfese, D.L. (2003). The role of environment in the development of reading skill: a longitudinal study of preschool and school-age measures. Journal of learning disabilities, 36 (1) 59-67.

National Research Council (2000). How People Learn: Brain, Mind, Experience, and School National Academy Press: Washington, D.C.

Oakhill, J.V. \& Cain, K. (2004) The development of comprehension skills. En T. Nunes y P.E. Bryant (Eds.), Handbook of Children's Literacy. Dordrect: Kluwer Academic Publisher.

Oliva, A. (2006). Relaciones familiares y desarrollo adolescente. Anuario de Psicología, 37, 209-223.

Oliva, A., Parra, A. y Arranz, A. (2008). Estilos relacionales materno y paterno y ajuste adolescente. Infancia y aprendizaje, 31 (1). 93-106.

Oliva, A., Parra, A, Sánchez-Queija, I. y López, F. (2007). Estilos educativos materno y paterno: evaluación y relación con el ajuste del adolescente. Anales de Psicología, 23 (1), 49-56.

Palacios, J. (1988). Las ideas de los padres sobre el desarrollo y la educación de sus hijos. Sevilla: Instituto de Desarrollo Regional.

Palacios, J., Marchesi, A. y Coll, C. (Eds.) (2004) Desarrollo Psicológico y Educación. 1. Psicología Evolutiva (pp. 103-132). Madrid: Alianza Editorial.

Pelegrina, S., Garcia, M. C. y Casanova, P. F. (2002). Los estilos educativos de los padres y la competencia académica de los adolescentes. Infancia y aprendizaje, 25(2), 147-168.

Peralbo, M. y Fernández, M.L.(2003) Estructura familiar y rendimiento escolar en educación secundaria obligatoria. Revista Galego-Portuguesa de Psicoloxía e educación, 7, 309322.

Pérez de Pablos, S. (2003) El papel de los padres en el éxito escolar de los hijos. Madrid.

Pérez-Díaz, V.; Rodríguez, J.C. y Sánchez Ferrer, L. (2001). La familia española ante la educación de sus hijos. Fundación La Caixa, Colección Estudios Sociales no 5.

PISA (2010) Programa para la evaluación internacional de alumnos de la OCDE. Informe Español Catálogo de publicaciones del MEC http://www.mec.es (consultado el 04/01/11).

Purcell-Gates, V. (1996). Stories, coupons, and the "TV Guide": Relationships between home literacy experiences and emergent literacy knowledge. Reading Research Quarterly, 31(4), 406-428. EJ 532191 
Ramos Sánchez, J.L. (2007). Evaluación de los procesos lectores en alumnos de tercer ciclo de Educación. PROLEC-SE. Madrid: TEA.

Rashid, F.L., Morris, R. D. y Sevick, R. A. (2005). Relationship between home literacy environment and reading achievement in children with reading disabilities. Journal of Learning Disabilities, 38, 2-11.

Silk, J., Morris, A., Kanaya, T. y Steinberg, L. (2003). Psychological Control and Autonomy Granting: Opposite Ends of a Continuum or Distinct Constructs?. Journal of Research on Adolescence, 13(1), 113-128.

Spear-Swerling, L. (2006) Children's reading comprehension and oral reading fluency in easy text. Reading and Writing, 19, 199-220.

Stanovich, K.E. \& West, R.F. (1989) Exposure to print and orthographic processing. Reading Research Quarterly, 24, 402-433.

Stattin, H. y Kerr, M. (2000). Parental monitoring: A reinterpretation. Child Development,71(4), 1072-1085.

Topping, K. J., Samuels, J. y Paul, D. P. (2007). Does practice make perfect? Independent reading quality, quantity and student achievement. Learning \& Instruction, 17, 253-264.

Whitehurst, G.J. y Lonigan, C.J. (1998). Child development and emergent literacy. Child Development, $69,848-872$

Wigfield, A., Eccles, J. S., McIver, D., Redman, D. y Medley, C. (1991). Transitions at early adolescence: Changes in children's domain-specific self-perceptions and general selfesteem across the transition to junior high school. Developmental Psychology, 27, 552565.

Young, W. J., Liner, M. R. y Brooks-Gunn, J. (2002). How money matters for young children's development: Parental investment and family processes. Child Development, 73, 1861-1879. 